\title{
Mesenchymal stem cell treatment in a critically ill COVID-19 patient: a case report
}

\author{
Rehile Zengin ${ }^{1}$, Oyku Beyaz ${ }^{2}$, Elif S. Koc ${ }^{2}$, Ibrahim O. Akinci ${ }^{3}$, Sesin Kocagoz ${ }^{1}$, Gulseren Sagcan ${ }^{4}$, \\ Ercument Ovali ${ }^{5}$, Caglar Cuhadaroglu ${ }^{4}$ \\ ${ }^{1}$ Department of Infectious Diseases, Acibadem Mehmet Ali Aydinlar University School of Medicine, Istanbul, Turkey; ${ }^{2}$ Department of Internal \\ Medicine, Acibadem Mehmet Ali Aydinlar University School of Medicine, Istanbul, Turkey; ${ }^{3}$ Department of Anesthesiology and Reanimation, \\ Acibadem Mehmet Ali Aydinlar University School of Medicine, Turkey; ${ }^{4}$ Department of Pulmonary Medicine, Acibadem Mehmet Ali Aydinlar \\ University School of Medicine, Istanbul, Turkey; ${ }^{5}$ Department of Hematology, Acibadem Labcell Laboratories, Acibadem Mehmet Ali Aydinlar \\ University School of Medicine, Istanbul, Turkey \\ Correspondence to: Dr. Oyku Beyaz. Department of Internal Medicine, Acibadem Mehmet Ali Aydinlar University School of Medicine, Acibadem \\ Altunizade Hastanesi, Yurtcan Sokak No.1 Uskudar 34662, Istanbul, Turkey. Email: oykubeyaz@gmail.com.
}

\begin{abstract}
An outbreak of a new coronavirus causing severe respiratory disease (COVID-19) was first reported in China and rapidly spread worldwide. Clinical spectrum changes from asymptomatic infection to severe illness and even death, and no specific treatment is currently available. A range of antiviral, antimalarial and antibiotic agents are being used. We report a case of a COVID-19 patient that progressed to severe disease requiring intubation and intensive care. We performed mesenchymal stem cell (MSC) transplantation considering the signs showing persistent excessive immune response and deterioration despite all supportive and drug therapies. The two rounds of transplantation did not result in any severe complications and was well-tolerated. Clinical signs were improved. The use of MSC therapy may be considered for compassionate use in selected patients.
\end{abstract}

Keywords: Coronavirus disease-2019 (COVID-19); mesenchymal stem cells (MSC); acute respiratory distress syndrome (ARDS); case report; stem cell treatment

Received: 28 May 2020; Accepted: 05 August 2020; Published: 08 September 2020.

doi: $10.21037 /$ sci-2020-024

View this article at: http://dx.doi.org/10.21037/sci-2020-024

\section{Introduction}

An outbreak of a new coronavirus [severe acute respiratory syndrome coronavirus (SARS-CoV-2)] that was first reported in China and rapidly spread worldwide, resulted in a pandemic (1-3). As of May 21, 2020, there were over 5 million cases and 320,000 deaths worldwide (4). The pathogen, having a zoonotic origin, later gained human-tohuman transmissibility via respiratory droplets or contact transmission (5). Patients with resulting infection named as coronavirus disease-2019 (COVID-19) usually present with fever, dry cough and shortness of breath and may quickly develop a severe acute respiratory distress syndrome (ARDS) (6).

The clinical spectrum can change from asymptomatic infection to severe illness and even death. It is reported that around $14 \%$ of reported cases developed severe disease and $5 \%$ were critically ill requiring intensive care (7). People at older ages and those with underlying health conditions including diabetes, hypertension, chronic obstructive pulmonary disease or immunosuppression are found to be more likely to develop severe outcomes (8). Although the main treatment is supportive and symptomatic, a range of antiviral agents, an anti-malarial (chloroquine) and antibiotics have been used to treat moderate and severe cases. Alternatives were also proposed such as convalescent plasma and mesenchymal stem cell (MSC) therapy for potential use in severe and critically ill patients to address disease progression via modifying immune response (9-11).

In this article, we report the case of a critically ill 

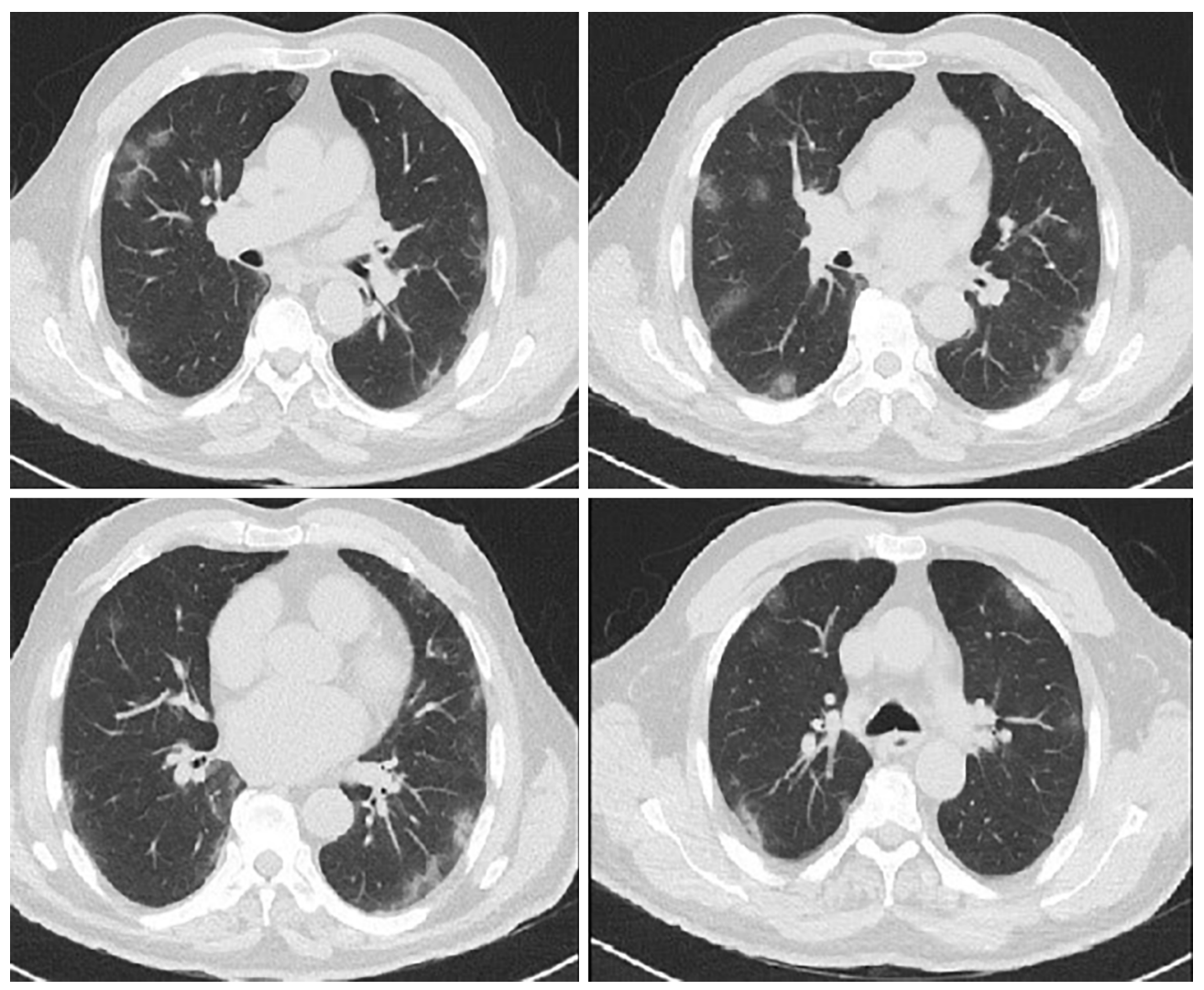

Figure 1 Thorax CT on admission.

COVID-19 patient received an experimental treatment with MSC therapy in conjunction with recommended treatment protocols. We present the following case in accordance with the CARE reporting checklist (available at http://dx.doi. org/10.21037/sci-2020-024).

\section{Case presentation}

A 72-year-old man with a history of hypertension, diabetes mellitus and hyperlipidemia presented to our emergency department on March 11, 2020, with fatigue, fever and dysphagia. The initial vital signs were within normal ranges. He had a temperature of $37.4{ }^{\circ} \mathrm{C}$. Physical examination revealed tonsillitis and aphthous stomatitis. Lung auscultation revealed diffuse bilateral rhonchi and chest radiograph showed low-density consolidation in both lungs. Laboratory tests showed thrombocytopenia (thrombocyte count: $88 \times 10^{3} / \mu \mathrm{L}$ ); normal leucocyte, lymphocyte and procalcitonin levels; and high levels of CRP $(4.6 \mathrm{mg} / \mathrm{dL})$. Given that he had returned from a trip to Italy 15 days ago before his symptoms has started, due to governmental requirement of that time, a throat swab was collected to perform COVID-19 test and comprehensive viral respiratory panel by polymerase chain reaction (PCR) [including Influenza A (H1, H1-2009, H3), Influenza B, Parainfluenza 1-2-3-4, RSV, Adenovirus, Coronavirus O34HKU-NL63-229E, MERS Coronavirus, Enterovirus, Rhinovirus, Parechovirus, Metapneumovirus, Mycoplasma pneumoniae, Chlamydophila penumoniae, Bordetella pertussis, Bordetella parapertussis, Pneumocystis jirovecii]. Viral respiratory panel test results were all negative. For further investigation, patient was admitted to the inpatient ward with a diagnosis of lower respiratory tract infection.

Atypical pneumonia PCR panel testing for Chlamydia, Mycoplasma and Legionella pneumonia, sputum culture and thorax CT were performed. Atypical pneumonia panel and sputum culture were negative. The CT revealed peripheral ground-glass opacities and reactive mediastinal lymphadenopathy, suggesting viral pneumonia (Figure 1). Levofloxacin $(1 \times 750 \mathrm{mg})$ and oseltamivir $(2 \times 75 \mathrm{mg})$ was initiated.

On day 5 of the hospitalization, the patient developed 


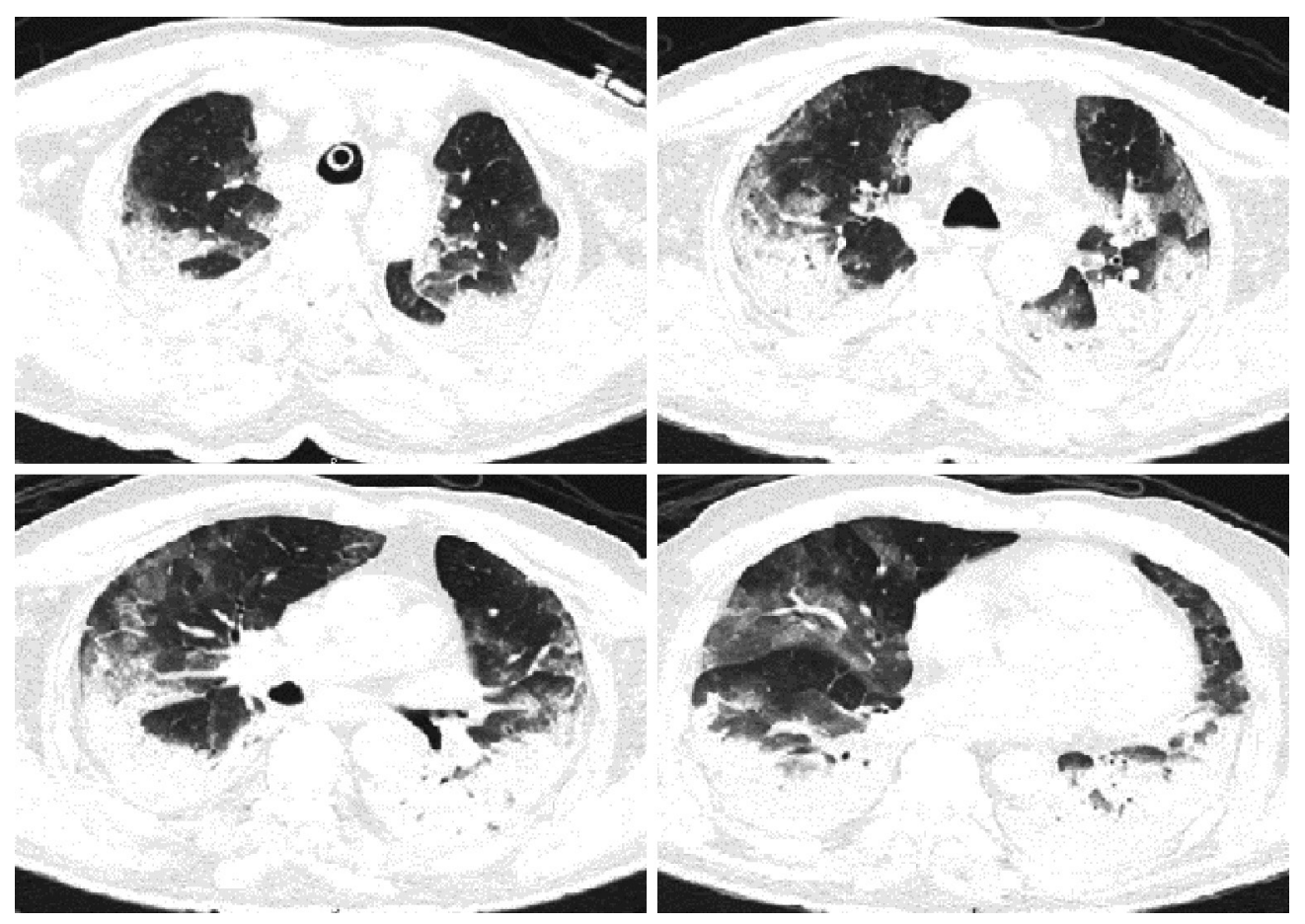

Figure 2 Thorax CT (day 6 of the hospitalization).

fever of $38.6{ }^{\circ} \mathrm{C}$ (axillary), required nasal oxygen $\left(\mathrm{O}_{2}\right)$ of $4 \mathrm{~L} / \mathrm{min}$, and had CRP level increased to $19.4 \mathrm{mg} / \mathrm{dL}$. Initial antibacterial therapy was replaced with piperacillin/ tazobactam and vancomycin until blood culture results are confirmed. Chloroquine and lopinavir/ritonavir were initiated with suspected COVID-19 in accordance with Turkish Ministry of Health's COVID-19 treatment guideline (12). In order to eliminate the potential risk of hemolytic anemia with the use of chloroquine, G6PD levels were tested and were found to be normal (G6PD: $12.6 \mathrm{IU} / \mathrm{g}$ $\mathrm{Hb})$. On the same day, throat swab was reported as positive for SARS-CoV-2 on real-time reverse-transcriptasepolymerase-chain-reaction (rRT-PCR) assay.

On day 6 , fever persisted at $39.6^{\circ} \mathrm{C}$, and the patient developed shortness of breath requiring oxygen supplementation $\left(\mathrm{sPO}_{2} 88 \%\right.$ with $\left.8 \mathrm{~L} / \mathrm{min}_{2}\right)$. Subsequent thorax CT showed atelectatic areas in the middle and lower parts of the lungs, in addition to the earlier image of ground-glass opacities and patchy infiltration (Figure 2). Candida albicans was isolated in sputum culture with more than 15 leukocytes, while other cultures such as blood and urine were negative. Comprehensive laboratory tests showed increased levels of D-Dimer $(1.12 \mathrm{mg} / \mathrm{L})$, ferritin (1,618 ng/mL), ALT (76 IU/L), AST (94 IU/L),
LDH (387 IU/L), BNP (899 pg/mL), PT (14.1 s) and normal levels of CK (52 IU/L), CK-MB $(0.430 \mathrm{ng} / \mathrm{mL})$, Troponin I $(0.026 \mathrm{ng} / \mathrm{mL})$ with low levels of albumin $(2.1 \mathrm{~g} / \mathrm{dL})$. Immunologic parameters were: CD4/CD8 ratio: 6.46, CD4 cell count: 951.46 cells $/ \mathrm{mm}^{3}$, CD8 cell count: 147.2 cells $/ \mathrm{mm}^{3}$. Interleukin-6 (IL-6) was high at: $181 \mathrm{pg} / \mathrm{mL}$ and tocilizumab $(1 \times 800 \mathrm{mg})$ was initiated. After administration of tocilizumab, temperature decreased rapidly.

Subsequently, the patient developed acute respiratory distress (ARDS) $\left(\mathrm{sPO}_{2} 86 \%\right.$ with $\left.15 \mathrm{~L} / \mathrm{min} \mathrm{O}_{2}\right)$, was intubated and admitted to the intensive care unit (ICU). Thorax CT showed progression of pneumonic consolidations and enlargement of the mediastinal lymph nodes. Second dose of tocilizumab were given on day 7 (1× $800 \mathrm{mg})$.

The patient who was hypoxemic, hypercapnic and progressively became hypotensive, was monitored with high-pressure mechanical ventilation (PEEP: $16 \mathrm{cmH}_{2} \mathrm{O}$, $\left.\mathrm{FiO}_{2}: 0.7, \mathrm{sPO}_{2}: 95 \%\right)$ and low-dose positive inotropic agents. Antibacterial therapy with piperacillin/tazobactam and vancomycin was continued with addition of empiric micafungin. Despite a decrease in CRP level $(2.49 \mathrm{mg} / \mathrm{dL})$, chest X-ray showed progression (Figure 3). Need for 


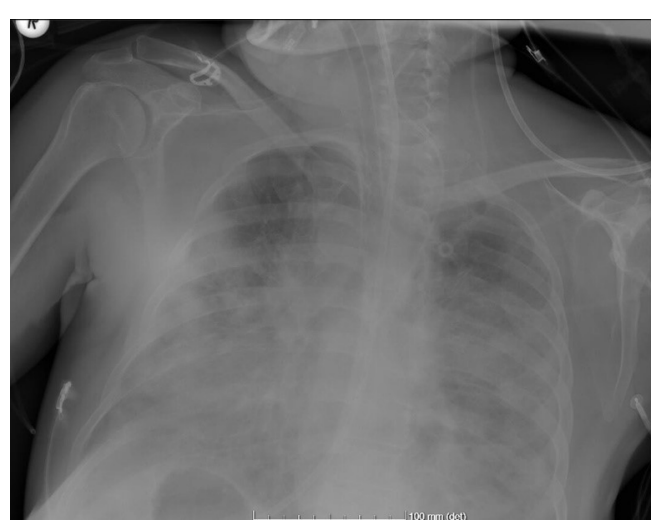

Figure 3 Anteroposterior chest radiograph (day 11 of the hospitalization).

inotropic support continued.

On day 12, laboratory exams showed higher levels of IL-6 (825 pg/mL), ALT (141 IU/L), AST (133 IU/L), LDH (575 IU/L), and reduced direct bilirubin $(0.24 \mathrm{mg} / \mathrm{dL})$, CD4/CD8 ratio (2.61). Absolute CD4 cell count was found to be consecutively getting lower $\left(416.40\right.$ cells $\left./ \mathrm{mm}^{3}\right)$. Considering these signs of severe inflammatory immune response and rapid deterioration of clinical course, along with his delayed arrival to our hospital, an investigational MSC transplantation was decided to be carried out and performed in intensive care unit, via intratracheal and intravenous routes $\left(0.7 \times 10^{6}\right.$ cells $/ \mathrm{kg}$ intravenous, $0.3 \times 10^{6}$ cells $/ \mathrm{kg}$ intratracheal with 4 unites of heparin). A second dose of MSC therapy $\left(0.7 \times 10^{6}\right.$ cells $/ \mathrm{kg}$ intravenous, $0.3 \times 10^{6}$ cells $/ \mathrm{kg}$ intratracheal routes with 4 unites of heparin) was given 5 days after the first dose while the patient was mechanically ventilated at PEEP: $6 \mathrm{cmH}_{2} \mathrm{O}$ with values of $\mathrm{FiO}_{2}: 0.4$ and $\mathrm{sPO}_{2}: 100$. The protocol for administration and the dosage was similar to that in the earlier published reports (10). No acute or delayed reactions or adverse effects were detected following administration of the treatment.

Umbilical cord-derived MSCs that were prepared at Acibadem Labcell Cellular Therapy Laboratory were used.

Approval from the Ministry of Health in the use of this therapy and informed consent from the close relatives of the patient were obtained.

Following the MSC transplantation, the need for inotropic agents started to disappear, acidosis, electrolyte imbalance and hypoxemia started to improve $\left(\mathrm{sPO}_{2}\right.$ was achieved $98 \%$ with adjusted $\mathrm{FiO}_{2}$ and PEEP levels of 0.6 and $14 \mathrm{cmH}_{2} \mathrm{O}$ respectively), and acute phase information markers such as CRP levels regressed $(0.4 \mathrm{mg} / \mathrm{dL})$. The patient continued to be in need of invasive ventilation for the next 5 days following the second dose. On day 19 of hospitalization, lung chest $\mathrm{X}$-ray showed slight regression in the ground-glass imaged infiltration in the middle right lung periphery, and significant regression in the lowdensity infiltrations in the lower right lung and lateral left lung. Chloroquine, lopinavir/ritonavir and vancomycin treatments were stopped.

The patient was extubated on day 22 and continued to be monitored in the ward, initially receiving nasal oxygen which was later discontinued. Thorax CT revealed regression in the infiltrations in both basal lobes, with ongoing pneumonia (Figure 4). The patient was discharged with planned chest physiotherapy and physical rehabilitation to restore myopathy. On his returns for routine follow-ups during the 2 months after the MSC transplantation, he had normal clinical and laboratory signs and no adverse effects due to the procedure were identified.

All procedures performed in studies involving human participants were in accordance with the Helsinki Declaration (as revised in 2013). Written informed consent was obtained from the patient for publication of this case report and any accompanying images. A copy of the written consent is available for review by the Editor-in-Chief of this journal.

\section{Discussion}

No specific treatment for COVID-19 is currently available. Although mild cases may only need supportive treatment, approved or experimental therapies are being used to manage especially severe cases. These include a combination therapy registered for use in HIV (lopinavir/ ritonavir), chloroquine which is used for prophylaxis and treatment of malaria, remdesivir and favipiravir, medications used in treatment of Ebola, and tocilizumab, an IL-6 receptor antagonist. Different combinations of these drugs are included in treatment protocols of countries and applied depending on the severity or stage of the infection (12-15).

Administration of mesenchymal stem cell therapy has been reported in a limited number of clinical settings in China. A study by Leng et al. involving seven patients received MSC treatment reported improving of functional outcomes. No acute infusion-related or other adverse reactions were observed during the time of the followup (10). Liang et al. described a critically ill case with normalization of vital, clinical and laboratory signs following the receipt of MSC therapy (16). 

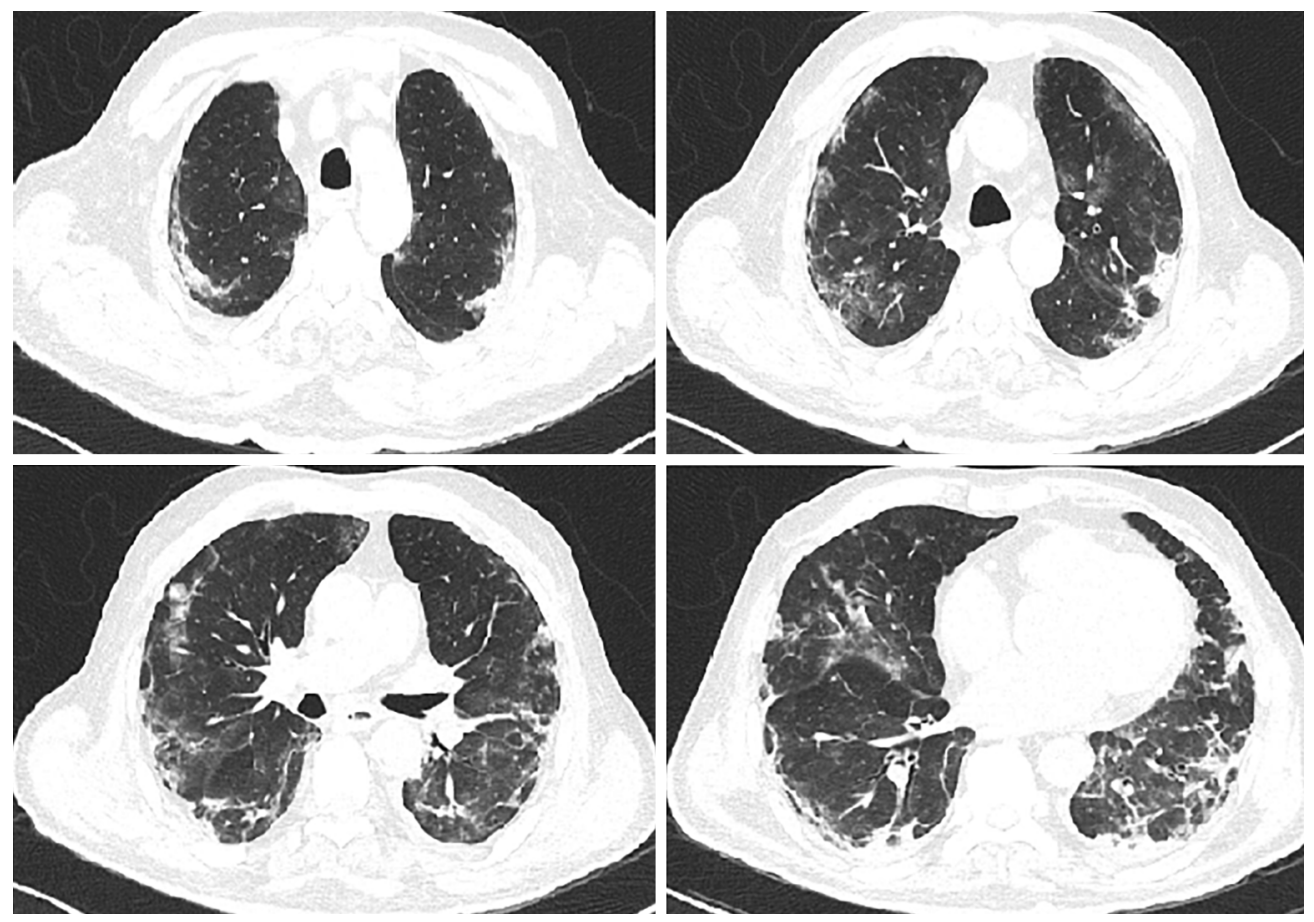

Figure 4 Thorax CT (day 30 of the hospitalization).

Mesenchymal stem cells have immunomodulatory, antimicrobial, anti-inflammatory and regenerative properties (17). They have been studied and used in a range of immune-mediated diseases, including graft-versus-host disease, type 1 diabetes mellitus, arthritis, and multiple sclerosis (18). Limited data from animal models have shown potential beneficial effects in virus-related pulmonary disease in some types of influenza viruses, but not in others $(19,20)$. Pre-clinical data from models for coronavirus respiratory infections is not currently available (20).

Possible effects of MSC in the treatment of respiratory disease may be due to: suppression of inflammatory processes by converting proinflammatory M1 macrophages into M2 macrophages, encoding macrophages through prostaglandin E2 secretion for increasing IL-10 production which is reduced in acute lung injury, reducing the lymphocyte response by inhibiting $\mathrm{T}$ cell proliferation and activation as well as regulating B cell functions (21); anti-proteolytic action inhibiting the protease activity that SARS-CoV-2 needs for transmission (18); ability to inhibit viral replication via siRNAs that are able to regulate gene expressions $(22,23)$; and antifibrotic properties, shown to reduce fibrosis in lung, heart, liver, and skin tissues (24).

A recent review noted that no significant issues in terms of safety of MSC administration in critically ill patients including those with ARDS resulting from other etiologies, have been reported to date. However, it also highlighted the limitation of evidence on efficacy, with only one unpublished exploratory trial of MSC administration showing beneficial outcomes (20).

Our case was a 72-year-old man, with chronic conditions and tested positive for COVID-19. Although natural course of the disease involves peaking at 7-14 days and improvement afterwards, our case was already on late stage on admission and rapidly progressed to severe disease despite all supportive and drug therapies. Signs showing continued excessive immune response, such as fever and very high levels of IL-6 prompted us to consider MSC therapy. Following administration of the treatment, no acute or delayed reactions or adverse effects were detected. Clinical signs were improved.

This case report is associated with several limitations. First, it is not possible to draw conclusions on treatment effects as it is a description of a single case. Second, although follow ups during the 2 months after the treatment revealed no adverse effects, the timeline of the study did not allow us to explore any potential longer-term side effects. However, the report has a number of strengths. Being a 
tertiary care center providing cutting edge medical and ICU care and designated as a pandemic hospital throughout COVID-19 outbreak, our hospital setting enabled the use of all necessary diagnostic, supportive and therapeutic interventions. Additionally, continuous and rigorous monitoring of clinical, laboratory and radiologic signs have ensured a thorough and systematic documentation of the progress.

In this case report, since MSC administration was supplemental to ongoing treatment of one single patient, we cannot conclude as to whether the natural course of COVID-19 with intensive care along with the use of drugs (including tocilizumab) recommended by the Turkish Ministry of Health's COVID-19 treatment guideline (12) or MSC transplantation has led to the clinical improvement. However, we show that the use of MSC therapy in our critically-ill case did not result in a severe outcome, it was well tolerated even in an intensive care setting and it may be considered for compassionate use in selected patients.

\section{Patient perspective}

"I was feeling very sick following a trip to Italy in early March. I went to the hospital and was hospitalized with a lung infection. The tests showed that I had the new virus. I was given medications and oxygen, but my condition did not improve, and after a while, I got worse. I could not breath. I was taken to intensive care. I do not remember my time there. Later when I woke up, I was feeling better. Now I am feeling much better and bave no complaints. I owe my life to the treatment I received."

The son of the patient who gave consent in the use of MSC treatment has reflected as: "My Dad became severely ill due to the new virus. He was one of the early patients at the beginning of the disease spread in Turkey. He was hospitalized and despite all medications and treatments, his condition worsened and was taken to intensive care. With no signs of improvement, I consented on using the stem cells as an investigative treatment. He is now in a very good condition, the infection has gone, and we are thankful. I believe be would have died if he had not received this treatment."

\section{Acknowledgments}

Funding: This work received funding from Acibadem Mehmet Ali Aydinlar University.

\section{Footnote}

Reporting Checklist: The authors have completed the CARE reporting checklist. Available at http://dx.doi.org/10.27/sci2020-024

Conflicts of Interest: All authors have completed the ICMJE uniform disclosure form (available at http://dx.doi. org/10.21037/sci-2020-024). The authors have no conflicts of interest to declare.

Ethical Statement: The authors are accountable for all aspects of the work in ensuring that questions related to the accuracy or integrity of any part of the work are appropriately investigated and resolved. All procedures performed in studies involving human participants were in accordance with the Helsinki Declaration (as revised in 2013). Written informed consent was obtained from the patient for publication of this case report and any accompanying images. A copy of the written consent is available for review by the Editor-in-Chief of this journal.

Open Access Statement: This is an Open Access article distributed in accordance with the Creative Commons Attribution-NonCommercial-NoDerivs 4.0 International License (CC BY-NC-ND 4.0), which permits the noncommercial replication and distribution of the article with the strict proviso that no changes or edits are made and the original work is properly cited (including links to both the formal publication through the relevant DOI and the license). See: https://creativecommons.org/licenses/by-nc-nd/4.0/.

\section{References}

1. Bedford J, Enria D, Giesecke J, et al. COVID-19: towards controlling of a pandemic. Lancet 2020;395:1015-8.

2. Del Rio C, Malani PN. COVID-19-New insights on a rapidly changing epidemic. JAMA 2020;323:1339-40.

3. World Health Organization. WHO Director-General's opening remarks at the media briefing on COVID-19. 11 March 2020. Available online: https://www.who.int/dg/ speeches/detail/who-director-general-s-opening-remarksat-the-media-briefing-on-covid-19---11-march-2020

4. Coronavirus Outbreak. Available online: https://www. worldometers.info/coronavirus/

5. Phan LT, Nguyen TV, Luong QC, et al. Importation and human-to-human transmission of a novel coronavirus in Vietnam. N Engl J Med 2020;382:872-4.

6. Huang C, Wang Y, Li X, et al. Clinical features of patients infected with 2019 novel coronavirus in Wuhan, China. Lancet 2020;395:497-506. 
7. Wu Z, McGoogan JM. Characteristics of and important lessons from the coronavirus disease 2019 (COVID-19) outbreak in China: Summary of a report of 72314 cases from the Chinese Center for Disease Control and Prevention. JAMA 2020;323:1239-42.

8. Chen T, Wu D, Chen H, et al. Clinical characteristics of 113 deceased patients with coronavirus disease 2019: retrospective study. BMJ 2020;368:m1091.

9. Shen C, Wang Z, Zhao F, et al. Treatment of 5 critically ill patients with COVID-19 with convalescent plasma. JAMA 2020;323:1582-9.

10. Leng Z, Zhu R, Hou W, et al. Transplantation of ACE2mesenchymal stem cells improves the outcome of patients with COVID-19 pneumonia. Aging Dis 2020;11:216-28.

11. Atluri S, Manchikanti L, Hirsch JA. Expanded umbilical cord mesenchymal stem cells (UC-MSCs) as a therapeutic strategy in managing critically ill COVID-19 patients: The case for compassionate use. Pain Physician 2020;23:E71-83.

12. TC Saglik Bakanligi (Turkish Ministry of Health). COVID-19 (SARS-CoV-2 enfeksiyonu) rehberi. Ankara: Ministry of Health; 2020. Available online: https:// covid19bilgi.saglik.gov.tr/depo/rehberler/COVID-19_ Rehberi.pdf

13. Colson P, Rolain J, Raoult D. Chloroquine for the 2019 novel coronavirus SARS-CoV-2. Int J Antimicrob Agents 2020;55:105923.

14. Mehta P, McAuley DF, Brown M, et al. COVID-19: consider cytokine storm syndromes and immunosuppression. Lancet 2020;395:1033-4.

15. Dong L, Hu S, Gao J. Discovering drugs to treat coronavirus disease 2019 (COVID-19). Drug Discov Ther 2020;14:58-60.

16. Liang B, Chen J, Li T, et al. Clinical remission of a critically ill COVID-19 patient treated by human umbilical cord mesenchymal stem cells. (ChinaXiv:202002.00084).

doi: $10.21037 /$ sci-2020-024

Cite this article as: Zengin R, Beyaz O, Koc ES, Akinci IO, Kocagoz S, Sagcan G, Ovali E, Cuhadaroglu C. Mesenchymal stem cell treatment in a critically ill COVID-19 patient: a case report. Stem Cell Investig 2020;7:17.
Available online: http://chinaxiv.org/abs/202002.00084

17. Shah TG, Predescu D, Predescu S. Mesenchymal stem cells-derived extracellular vesicles in acute respiratory distress syndrome: a review of current literature and potential future treatment options. Clin Transl Med 2019;8:25.

18. El Haddad N, Heathcote D, Moore R, et al. Mesenchymal stem cells express serine protease inhibitor to evade the host immune response. Blood 2011;117:1176-83.

19. Cardenes N, Aranda-Valderrama P, Carney JP, et al. Cell therapy for ARDS: efficacy of endobronchial versus intravenous administration and biodistribution of MAPCs in a large animal model. BMJ Open Respir Res 2019;12;6:e000308.

20. Khoury M, Cuenca J, Cruz FF, et al. Current status of cell-based therapies for respiratory virus infections: Applicability to COVID-19. Eur Respir J 2020;55:2000858.

21. Abumaree MH, Al Jumah MA, Kalionis B, et al. Human placental mesenchymal stem cells play a role as immune suppressive cells by shifting macrophage differentiation from inflammatory M1 to anti-inflammatory M2 macrophages. Stem Cell Rev Rep 2013;9:620-41.

22. Chan MCW, Kuok DIT, Leung CYH, et al. Human mesenchymal stromal cells reduce influenza A H5N1associated acute lung injury in vitro and in vivo. Proc Natl Acad Sci U S A 2016;113:3621-6.

23. Alcayaga-Miranda F, Cuenca J, Khoury M. Antimicrobial activity of mesenchymal stem cells: Current status and new perspectives of antimicrobial peptide-based therapies. Front Immunol 2017;8:339.

24. Dong LH, Jiang YY, Liu YJ, et al. The anti-fibrotic effects of mesenchymal stem cells on irradiated lungs via stimulating endogenous secretion of HGF and PGE2. Sci Rep 2015;5:8713. 